\title{
STABILITY OF ANOSOV MAPS
}

\author{
JUERGEN QUANDT
}

(Communicated by Kenneth R. Meyer)

\begin{abstract}
Anosov maps of Banach manifolds are shown to be inverse limit
\end{abstract} stable.

It is well known that D. Anosov [1] introduced a class of diffeomorphisms of compact manifolds, whose elements he showed to be structurally stable. Using the concept of inverse limit stability, R. Mané and C. Pugh [2] and F. Przytycki [3] have extended this result to nonsingular maps of compact manifolds.

In the present paper we prove a further extension by allowing singularities and Banach manifolds. We show the inverse limit stability of compact hyperbolic invariant sets and, as a consequence, the inverse limit stability and openness of Anosov maps. A similar discussion for semiflows may be found in [4].

0. Inverse limit stability. Let $X$ be a Banach manifold with a complete Riemannian structure, and $d$ the corresponding metric on $X$.

The class of all $C^{1}$-maps of $X$ will be denoted by $\operatorname{End}^{1}(X)$, and equipped with the quasimetric

$$
\sup \left\{d\left(D^{i} f(x), D^{i} g(x)\right)|| x \in X \text { and } i \in\{0,1\}\right\} .
$$

With $X^{\mathbf{Z}}$ we denote the class of all maps from $\mathbb{Z}$ to $X$, and choose

$$
\tilde{d}(v, w)=\sum_{i=-\infty}^{+\infty} 2^{-|i|} d(v(i), w(i)) \quad \text { for } v, w \in X^{\mathbf{Z}}
$$

as a corresponding quasimetric.

Any map $f: X \rightarrow X$ induces a map $\tilde{f}: X^{\mathbf{Z}} \rightarrow X^{\mathbf{Z}}$ through

$$
\tilde{f}(v)=f v \quad \text { for } v \in X^{\mathbf{Z}}
$$

called the lift of $f$ to $X^{\mathbf{Z}}$.

Given an $\omega$-invariant set $I(f)$ for $f$, i.e., $f[I(f)] \subseteq I(f)$, we define

$$
\widetilde{I(f)}=\left\{v \in I(f)^{\mathbf{Z}} \mid f(v(i))=v(i+1) \text { for all } i \in \mathbb{Z}\right\},
$$

that is the set of all global sequences of $f$ with values in $I(f)$,

$$
S(\tilde{f})=\left\{v \in X^{\mathbf{Z}} \mid f(v(i))=v(i+1) \text { for all } i \in \mathbb{Z}\right\},
$$

that is the set of all global sequences of $f$, and

$$
A(f)=\bigcap_{n \geq 0} f^{n}[X]
$$

Received by the editors June 16, 1987.

1980 Mathematics Subject Classification (1985 Revision). Primary 58F10; Secondary 35F15. 
that is the set of values in $X$, through which a global sequence of $f$ passes.

This last remark is clear from the following lemma.

Let $p_{j}: X^{\mathbf{Z}} \rightarrow X, j \in \mathbb{Z}$, denote the $j$ th projection.

LEMMA 1. Let $f \in \operatorname{End}^{1}(X)$. Then the diagram

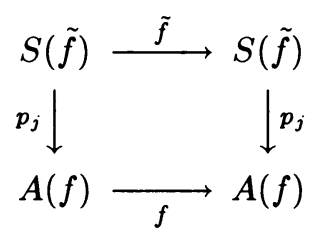

commutes for all $j \in \mathbb{Z}$ and $p_{j}[S(\tilde{f})]=A(f)$. Moreover, $\tilde{f}$ is bijective on $S(\tilde{f})$.

Proof. Easy.

We notice that $X^{\mathbf{Z}}$ may not be a manifold (in the usual sense). However, we can define tangent spaces and tangent maps as follows.

For $v, w \in X^{\mathbf{Z}}$ and $f \in \operatorname{End}^{1}(X)$, we set

$$
T_{v} X^{\mathbf{Z}}=\stackrel{+\infty}{\times} T_{v(i)} X
$$

and let $T_{v} \tilde{f}: T_{v} X^{\mathbf{Z}} \rightarrow T_{\tilde{f}(v)} X^{\mathbf{Z}}$ be given by

$$
T_{v} \tilde{f}(w)=\stackrel{+\infty}{\times} T_{v(i)} f(w(i)) .
$$

As in [2, 3] we will say that two $C^{\mathbf{1}}$-maps $f$ and $g$ of $X$ are inverse limit conjugate if there exists a homeomorphism $H: S(\tilde{f}) \rightarrow S(\tilde{g})$ such that $H \tilde{f}=\tilde{g} H$ on $S(\tilde{f})$. A $C^{1}$-map $f$ of $X$ is called $C^{1}$-inverse limit stable if there exists a $C^{1}$-neighborhood $U$ of $f$ in $\operatorname{End}^{1}(X)$ such that for every $g \in U$, the maps $f$ and $g$ are inverse limit conjugate. We notice that inverse limit stability compares global sequences, i.e., elements of $X^{\mathbf{Z}}$, rather than orbits, i.e., elements of $X$. Moreover, the concept of inverse limit stability for $C^{1}$-maps is a natural extension of the concept of structural stability for diffeomoprhisms, i.e., for diffeomorphisms the two concepts coincide. Fixed points, periodic points, and the asymptotic behavior of global sequences are preserved under this form of stability.

The following two technical lemmas are quite useful in proving inverse limit stability.

LEMMA 2. Let $f, g \in \operatorname{End}^{1}(X)$. If there exists a map $H: S(\tilde{f}) \rightarrow X^{\mathbf{Z}}$ making the following diagram commute

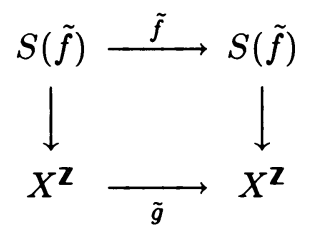

then there exists an "adjusted" map $\widehat{H}: S(\tilde{f}) \rightarrow X^{\mathbf{Z}}$ defined through $p_{j} \widehat{H}(v)=$ $p_{0} H(v(\cdot+j))$ for all $v \in S(\tilde{f})$ and $j \in \mathbb{Z}$, which also commutes the above diagram 
and, in addition, satisfies $\widehat{H}[S(\tilde{f})] \subseteq S(\tilde{g})$. (We give a simple example which shows that this last assumption is not automatically true for $H$.)

PrOOF. Let $H$ and $\widehat{H}$ be as above. Clearly the following diagram commutes:

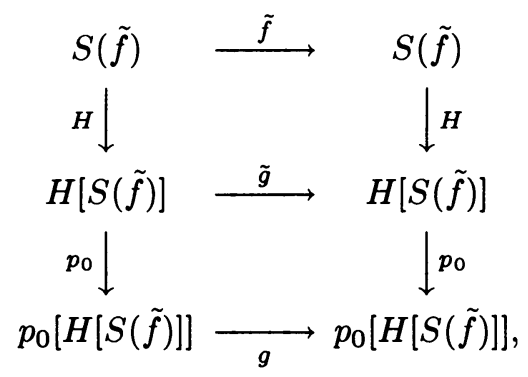

i.e., we have $g p_{0} H=p_{0} H \tilde{f}$. We then must show that (1) $\widehat{H}[S(\tilde{f})] \subseteq S(\tilde{g})$, and (2) $\widehat{H} \tilde{f}(v)=\tilde{g} \hat{H}(v)$ for all $v \in S(\tilde{f})$.

Let $v \in S(\tilde{f})$ and $i, j \in \mathbb{Z}$. Then

$$
\begin{aligned}
g\left(p_{i}(\widehat{H}(v))\right) & =g\left(p_{0}(H(v(\cdot+i)))\right)=g\left(p_{0}\left(H\left(\tilde{f}^{i}(v)\right)\right)\right) \\
& =p_{0}\left(H\left(\tilde{f}\left(\tilde{f}^{i}(v)\right)\right)\right)=p_{0}\left(H\left(\tilde{f}^{i+1}(v)\right)\right) \\
& =p_{0}(H(v(\cdot+i+1)))=p_{i+1}(\hat{H}(v)), \\
p_{j}(\hat{H}(\tilde{f}(v))) & =p_{j}(\hat{H}(v(\cdot+i)))=p_{0}(H(v(\cdot+i+j))),
\end{aligned}
$$

and

$$
\begin{aligned}
p_{j}(\tilde{g}(\widehat{H}(v))) & =g\left(p_{j}(\widehat{H}(v))\right)=g\left(p_{0}(H(v(\cdot+j)))\right) \\
& =p_{0}(H(v(\cdot+i+j))) . \quad \square
\end{aligned}
$$

To see that not every commuting map $H$ satisfies $H[S(\tilde{f})] \subseteq S(\tilde{g})$, we consider $X=\{0,1\}$ and $f \equiv g \equiv$ id. Then $S(\tilde{f})=S(\tilde{g})=\{(\ldots 000 \ldots),(\ldots \cdot 111 \ldots)\}$, and if $H: S(\tilde{f}) \rightarrow X^{\mathbf{Z}}$ is the map taking $(\ldots 000 \ldots)$ and $(\ldots 111 \ldots)$ into $(\ldots 0011 \ldots)$, it follows that $H \tilde{f}=\tilde{g} H$ on $S(\tilde{f})$, but $H[S(\tilde{f})]=\{(\ldots 0011 \ldots)\} \nsubseteq S(\tilde{g})$. The adjusted conjugacy $\widehat{H}$, in this case, takes $(\ldots 000 \ldots)$ and $(\ldots \cdot 11 \ldots)$ into $(\ldots \cdot 000 \ldots)$.

LEMMA 3. Let $H$ and $\hat{H}$ be as above.

(i) If $H$ is continuous, then so is $\hat{H}$.

(ii) If $H$ is close to the identity of $X^{\mathbf{Z}}$, then so is $\hat{H}$.

ProOF. (i). Let $v_{n} \rightarrow v$ in $S(\tilde{f})$, and hence

$$
v_{n}(\cdot+j) \rightarrow v(\cdot+j) \quad \text { in } S(\tilde{f}) \text { for all } j \in \mathbb{Z} .
$$

To see that $\hat{H}\left(v_{n}\right) \rightarrow \hat{H}(v)$ in $X^{\mathbf{Z}}$ it suffices to show that

$$
p_{j} \hat{H}\left(v_{n}\right) \rightarrow p_{j} \hat{H}(v) \quad \text { in } X \text { all } j \in \mathbb{Z}
$$

or equivalently that

$$
p_{0} H\left(v_{n}(\cdot+j)\right) \rightarrow p_{0} H(v(\cdot+j)) \quad \text { in } X \text { all } j \in \mathbb{Z} .
$$


But this is clear, since $p_{0}$ and $H$ are continuous.

(ii). Let $\mu>0$ (small) and suppose that

$$
\sum_{i=-\infty}^{+\infty} 2^{-|i|} d\left(p_{i}(v), p_{i} H(v)\right)<\mu \quad \text { for all } v \in S(\tilde{f}) .
$$

We want to show that

$$
\sum_{j=-\infty}^{+\infty} 2^{-|j|} d\left(p_{j}(v), p_{j} \hat{H}(v)\right)<4 \mu \text { for all } v \in S(\tilde{f}) .
$$

But since with $v \in S(\tilde{f})$ also $v(\cdot+j) \in S(\tilde{f})$ for $j \in \mathbb{Z}$, we have

$$
\sum_{i=-\infty}^{+\infty} 2^{-|i|} d\left(p_{i}(v(\cdot+j)), p_{i} H(v(\cdot+j))\right)<\mu \quad \text { for all } v \in S(\tilde{f}) \text { and } j \in \mathbb{Z}
$$

and hence

$$
d\left(p_{j}(v), p_{0} H(v(\cdot+j))\right)<\mu \quad \text { for all } v \in S(\tilde{f}) \text { and } j \in \mathbb{Z} .
$$

1. Anosov maps. As in $[2,3]$ we will say that a $C^{1}$-map $f$ of $X$ has a (weak) hyperbolic structure for an $\omega$-invariant set $I(f)$ if, for every $v \in \widetilde{I(f)}$, there exists a splitting of $\bigcup_{i=-\infty}^{+\infty} T_{v(i)} X$ into a direct sum $X^{s} \oplus X^{u}$, (varying continuously with $v \in \widetilde{I(f)})$, where

$$
\begin{gathered}
X^{s}=\bigcup_{i=-\infty}^{+\infty} X_{v(i)}^{s} \quad \text { and } X^{u}=\bigcup_{i=-\infty}^{+\infty} X_{v(i)}^{u}, \\
T_{v(i)} f\left[X_{v(i)}^{s}\right] \subseteq X_{v(i+1)}^{s} \quad \text { and } T_{v(i)} f\left[X_{v(i)}^{u}\right] \subseteq X_{v(i+1)}^{u}
\end{gathered}
$$

for all $i \in \mathbb{Z}$, and there are constants $c>0$ and $0 \leq \mu<1$ such that

$$
\begin{gathered}
\left|\left(T_{v(i)} f\right)^{n}(x)\right| \leq c \mu^{n}|x| \quad \text { for all } i \in \mathbb{Z}, n \in \mathbf{N}_{+} \text {and } x \in X_{v(i)}^{s}, \\
\left|\left(T_{v(i)} f\right)^{n}(x)\right| \geq c^{-1} \mu^{-n}|x| \quad \text { for all } i \in \mathbb{Z}, n \in \mathbf{N}_{+} \text {and } x \in X_{v(i)}^{u} .
\end{gathered}
$$

We notice that the definition of a (weak) hyperbolic structure does not imply a splitting of the whole restricted tangent bundle of $X$ over $I(f)$. Moreover, the map $f$ has a (weak) hyperbolic structure for $I(f)$ precisely if it has a (weak) hyperbolic structure for $\bigcap_{n \geq 0} f^{n}[I(f)]$. In case that the (weak) hyperbolic set is $A(f)$ and $A(f)$ is compact in $X$, we call $f$ an Anosov map.

We now turn to the proof of the inverse limit stability of Anosov maps. Since we allow singularities, we use a different approach than in [2, 3]. Our basic idea is to translate properties of a map $f \in \operatorname{End}^{1}(X)$ to its lift $\tilde{f}$, and to use this map's bijectiveness on $S(\tilde{f})$.

LEMMA 4. Let $f \in \operatorname{End}^{1}(X)$ and assume that $f$ has a (weak) hyperbolic structure for an $\omega$-invariant set $I(f)$. Then $\tilde{f}$ has a (weak) hyperbolic structure for $\widetilde{I(f)}$.

ProOF. Let $v \in \widetilde{I(f)}$. For $w \in T_{v} X^{\mathbf{Z}}$ we set $\|w\|=\sum_{i=-\infty}^{+\infty} 2^{-|i|}|w(i)|$, and define

$$
X_{v(\cdot+j)}^{\mathbf{Z}^{s}}=\stackrel{+\infty}{\times}_{i=-\infty}^{+\infty} X_{v(i+j)}^{s} \text { and } \quad X_{v(\cdot+j)}^{\mathbf{Z}^{u}}=\underset{i=-\infty}{+\infty} X_{v(i+j)}^{u}
$$


Since $f$ has a (weak) hyperbolic structure for $I(f)$, it follows that there exists a splitting of $\bigcup_{j=-\infty}^{+\infty} T_{v(\cdot+j)} X^{\mathbf{Z}}$ into a direct sum $X^{\mathbf{Z}^{s}} \oplus X^{\mathbf{Z}^{u}}$, where

$$
\begin{aligned}
& X^{\mathbf{Z}^{s}}=\bigcup_{j=-\infty}^{+\infty} X_{v(\cdot+j)}^{\mathbf{Z}^{s}} \text { and } X^{\mathbf{Z}^{u}}=\bigcup_{j=-\infty}^{+\infty} X_{v(\cdot+j)}^{\mathbf{Z}^{u}} \text {, } \\
& T_{v(\cdot+j)} \tilde{f}\left[X_{v(\cdot+j)}^{\mathbf{Z}^{s}}\right] \subseteq X_{v(\cdot+j+1)}^{\mathbf{Z}^{s}} \quad \text { and } \quad T_{v(\cdot+j)} \tilde{f}\left[X_{v(\cdot+j)}^{\mathbf{Z}^{u}}\right] \subseteq X_{v(\cdot+j+1)}^{\mathbf{Z}^{u}}
\end{aligned}
$$

for all $j \in \mathbf{Z}$.

Moreover, we have

$$
\begin{aligned}
\left\|\left(T_{v} \tilde{f}\right)^{n}(w)\right\| & =\sum_{i=-\infty}^{\infty} 2^{-|i|}\left|\left(T_{v(i)} f\right)^{n}(w(i))\right| \\
& \leq c \mu \sum_{i=-\infty}^{+\infty} 2^{-|i|}|w(i)| \\
& =c \mu\|w\|
\end{aligned}
$$

for all $n \in \mathbf{N}_{+}$and $w \in X_{v}^{\mathbf{Z}^{s}}$, and analogously

$$
\left\|\left(T_{v} \tilde{f}\right)^{n}(w)\right\| \geq c^{-1} \mu^{-n}\|w\|
$$

for all $n \in \mathbf{N}_{+}$and $w \in X_{v}^{\mathbf{Z}^{u}}$.

LEMMA 5. Let $f \in \operatorname{End}^{1}(X)$ and let $I(f)$ be an $\omega$-invariant set for $f$. If $\bigcap_{n \geq 0} f^{n}[I(f)]$ is compact in $X$, then $\widetilde{I(f)}$ is compact in $X^{\mathbf{Z}}$.

ProOF. As in Lemma 1 we find that $p_{j}[\widetilde{I(f)}]=\bigcap_{n \geq 0} f^{n}[I(f)]$ for $j \in \mathbf{Z}$. Thus we can write

$$
\widetilde{I(f)}=\left\{v \in\left(\bigcap_{n \geq 0} f^{n}[I(f)]\right)^{\mathbf{Z}} \mid f(v(i))=v(i+1) \text { for all } i \in \mathbf{Z}\right\}
$$

and Tychonov's theorem implies that $\left(\bigcap_{n \geq 0} f^{n}[I(f)]\right)^{Z}$ is compact in the subset topology. Thus it remains to show that $\widetilde{I(f)}$ is closed in $\left(\bigcap_{n \geq 0} f^{n}[I(f)]\right)^{Z}$. We denote the restricted projections to $\left(\bigcap_{n \geq 0} f^{n}[I(f)]\right)^{\mathbf{Z}}$ again by $p_{j}$, and assume that $v \in\left(\bigcap_{n \geq 0} f^{n}[I(f)]\right)^{\mathbf{Z}} \backslash \widetilde{I(f)}$, i.e., there is an $i \in \mathbf{Z}$ with $f(v(i)) \neq v(i+1)$. Thus, by continuity of $f$, there exists an $\varepsilon_{1}$-neighborhood $U$ of $v(i)$ in $\bigcap_{n \geq 0} f^{n}[I(f)]$ and an $\varepsilon_{2}$-neighborhood $V$ of $v(i+1)$ in $\bigcap_{n \geq 0} f^{n}[I(f)]$ such that $f[U] \cap V=\varnothing$. Setting $\varepsilon=\min \left\{2^{-|i|} \varepsilon_{1}, 2^{-|i+1|} \varepsilon_{2}\right\}$, we see that $p_{i}^{-1}[U] \cap p_{i+1}^{-1}[V]$ is an $\varepsilon$-neighborhood of $v$ in $\left(\bigcap_{n \geq 0} f^{n}[I(f)]\right)^{\mathbf{Z}}$, which does not meet $\widetilde{I(f)}$.

We can now prove the inverse limit stability of compact (weak) hyperbolic invariant sets.

PROPOSITION 1. Let $f \in \operatorname{End}^{1}(X)$ and let $I(f)$ be an $\omega$-invariant set for $f$. Suppose that $f$ has a (weak) hyperbolic structure for $I(f)$ and that $\bigcap_{n \geq 0} f^{n}[I(f)]$ is compact in $X$. Then there exists a $C^{1}$-neighborhood $U$ of $f$ in $\operatorname{End}^{1}(X)$ such that for 
all $g \in U$ there is an $\omega$-invariant set $J(g)$ for $g$ and a homeomorphism $H: \widetilde{I(f)} \rightarrow$ $\widetilde{J(g)}$ with $H \tilde{f}=\tilde{g} H$ on $\widetilde{I(f)}$. By choosing the neighborhood $U$ sufficiently small we will have $H$ arbitrarily close to the identity. Subject to this restriction, the conjugacy $H$ is unique.

PROOF. Let $C^{0}\left(\widetilde{I(f)}, X^{\mathbf{Z}}\right)$ denote the set of all continuous functions from $\widetilde{I(f)}$ into $X^{\mathbf{Z}}$, equipped with the quasimetric $d_{0}(F, G)=\sup \{d(F(v), G(v)) \mid v \in$ $\widetilde{I(f)}\}$ for $F, G \in C^{0}\left(\widetilde{I(f)}, X^{\mathbf{Z}}\right)$. Then consider the function $\tilde{f}^{\#}: C^{0}\left(\widetilde{I(f)}, X^{\mathbf{Z}}\right) \rightarrow$ $C^{0}\left(\widetilde{I(f)}, X^{\mathbf{Z}}\right)$ defined by $\tilde{f}^{\#}(H)=\tilde{f} H \tilde{f}_{\mid \widetilde{I}(f)}^{-1}$. This map has a fixed point at the inclusion map $i_{\tilde{f}}: \widetilde{I(f)} \rightarrow X^{\mathbf{Z}}$, taking $v$ into $v$. Since $\tilde{f}$ has a (weak) hyperbolic structure for $\widetilde{I(f)}$ by Lemma 3 , it follows that $i_{\tilde{f}}$ is a hyperbolic fixed point. If $g$ is $C^{1}$-near $f$, then $\tilde{g}$ is $C^{1}$-near $\tilde{f}$ with respect to the corresponding quasimetric on $\operatorname{End}^{1}\left(X^{\mathbf{Z}}\right)$. This implies that $\tilde{g}^{\#}: C^{0}\left(\widetilde{I(f)}, X^{\mathbf{Z}}\right) \rightarrow C^{0}\left(\widetilde{I(f)}, X^{\mathbf{Z}}\right)$, the map taking $H$ into $\tilde{g} H \tilde{f}_{\mid \tilde{I}(f)}^{-1}$, is $C^{1}$-near $\tilde{f}^{\#}$, and has a unique fixed point $H$, which is $C^{0}$-near $i_{\tilde{f}}$. We then observe that $\tilde{f}_{\mid \widetilde{I}(f)}$ is expansive, i.e., there exists a constant $\varepsilon>0$ such that, if $v, w \in \widetilde{I(f)}$ with $\tilde{d}\left(\tilde{f}^{n}(v), \tilde{f}^{n}(w)\right) \leq \varepsilon$ for all integers $n$, then $v=w$. This is a local result and follows as in Proposition 2 of [2]. Expansiveness implies that $H$ is one-to-one, provided the $C^{0}$-distance of $H$ from $i_{\tilde{f}}$ is less than $\frac{1}{2} \varepsilon$. Namely, let $v, w \in \widetilde{I(f)}$ and suppose that $H(v)=H(w)$. Then

$$
H \tilde{f}(v)=\tilde{g} H(v)=\tilde{g} H(w)=H \tilde{f}(w),
$$

which implies

$$
\begin{aligned}
\tilde{d}\left(\tilde{f}^{n}(v), \tilde{f}^{n}(w)\right) \leq \tilde{d}\left(\tilde{f}^{n}(v), H \tilde{f}^{n}(v)\right)+\tilde{d}\left(H \tilde{f}^{n}(v), H \tilde{f}^{n}(w)\right) \\
\\
+\tilde{d}\left(H \tilde{f}^{n}(w), \tilde{f}^{n}(w)\right) \leq \frac{1}{2} \varepsilon+0+\frac{1}{2} \varepsilon=\varepsilon
\end{aligned}
$$

for all $n \in \mathbb{Z}$, and hence $v=w$. Since $\widetilde{I(f)}$ is compact in $X^{\mathbf{Z}}$ by Lemma 4 , it follows that $H: \widetilde{I(f)} \rightarrow H[\widetilde{I(f)}]$ is a homeomorphism. To see that its range is contained in $S(\tilde{g})$, we use Lemmas 1 and 2 . They show that the "adjusted" map $\widehat{H}$ also commutes $\tilde{f}$ and $\tilde{g}$ on $\widetilde{I(f)}$ and satisfies $\widehat{H}[\widetilde{I(f)}] \subseteq S(\tilde{g})$. Uniqueness then implies that $H=\widehat{H}$. We therefore may choose $J(g)=p_{0}[H(\widetilde{I(f)}]]$.

Let us now make the following two observations.

First, the map $g$ has a (weak) hyperbolic structure for $J(g)$, because the set of hyperbolic linear maps of a Banach space $E$ is open in the set of all continuous linear maps of $E$.

Secondly, if $I(f)$ is equal to $A(f)$, i.e., $I(f)$ is the maximal compact invariant set for $f$, then the range of the above conjugacy is equal to $S(\tilde{g})$.

This leads to the following theorem.

THEOREM 1. Anosov maps of $X$ are $C^{1}$-inverse limit stable and form an open subset in $\operatorname{End}^{1}(X)$.

Examples of such maps, which may have singularities, can be constructed on product manifolds. Let, for example, $M$ and $N$ be compact manifolds and $f=$ $p\left(\operatorname{id}_{\mid M} g\right)$, where $p: M \times N \rightarrow * \times N$ is the projection, and $g: N \rightarrow N$ is an Anosov diffeomorphism. Then $f$ is a "singular" Anosov map. 
ACKNOwledgment. I would like thank Professors Jack Hale, Zbigniew Nitecki, and Michael Shub for their help.

\section{REFERENCES}

1. D. Anosov, Geodesic flows on compact Riemannian manifolds of negative curvature, Trudy Mat. Inst. Steklov 90 (1967) = Proc. Steklov. Inst. Math. 90 (1967).

2. R. Mané and C. Pugh, Stability of endomorphisms, Dynamical Systems-Warwick 1974 (Proc. Sympos., Univ. Warwick, Coventry 1973/74), Lecture Notes in Math., Vol. 468, SpringerVerlag, Berlin and New York, 1975, pp. 175-184.

3. F. Przytycki, Anosov endomorphisms, Studia Math. 58 (1976), 249-285.

4. J. Quandt, On structural stability for semiflows, Comm. Math. Phys. 117 (1988), 191-202.

Department of Mathematics, Columbia University, New York, New York 10027 